\title{
If you could see what we see, would it bother you?
}

Nadine C. Kassis $\mathrm{MD}^{1}$, Jennifer J. Hamner $\mathrm{DO}^{2}$, Michelle M. Takase-Sanchez $\mathrm{MD}^{3}$, Waseem Khoder $\mathrm{MD}^{3}$, Douglass S. Hale $\mathrm{MD}^{3}$, Michael H. Heit MD, $\mathrm{PhD}^{3}$

1 Toledo Clinic Urogynecology, Toledo, Ohio

2 Indiana University School of Medicine, Department of Obstetrics and Gynecology, Indianapolis, Indiana

3 Indiana University Health/Methodist Hospital, Urogynecology Associates, Indianapolis, Indiana

Corresponding Author: Nadine C. Kassis, MD

Toledo Clinic Urogynecology, 3425 Executive Parkway, Suite 108, Toledo, OH 43606

Phone: 419-214-3192, Fax: 419-214-3195, Email: nkassis@toledoclinic.com

Funding: None

Disclosures: Dr. Hale is a consultant with Up to Date and President of AUGS. Dr. Sanchez is a consultant for Pelvalon. The remaining authors have no disclosures or conflicts of interest.

Presentations: Oral presentation at the American Urogynecologic Society $36^{\text {th }}$ Annual Scientific Meeting, October 2015, Seattle, Washington

Author Roles: Kassis - study design, recruitment, database entry, data analysis, manuscript writing and editing; Hamner - database entry, data analysis, manuscript editing; Sanchez recruitment, manuscript editing; Khoder - recruitment, manuscript editing; Hale - study design, manuscript editing; Heit - study design, recruitment, data analysis, manuscript writing and editing

Abstract Word Count/Manuscript Body Word Count: 226/3057

This is the author's manuscript of the article published in final edited form as: Kassis, N. C., Hamner, J. J., Takase-Sanchez, M. M., Khoder, W., Hale, D. S., \& Heit, M. H. (2016). If you could see what we see, would it bother you? International Urogynecology Journal, 1-6. https://doi.org/10.1007/s00192-016-3073-x 


\begin{abstract}
Objective: The purpose of our study is to determine if the anatomic threshold for pelvic organ prolapse diagnosis and surgical success remains valid when the patient sees what we see on exam.
\end{abstract}

Methods: 200 participants were assigned, by computer generated block randomization, to see one of 4 videos. Each video contained the same six clips representative of various degrees of anterior vaginal wall support. Participants were asked questions immediately after each clip. They were asked: "In your opinion, does this patient have a bulge or something falling out that she can see or feel in the vaginal area". Similarly, they were asked to give their opinion on surgical outcome on a 4-point Likert scale.

Results: The proportion of participants who identified the presence of a vaginal bulge increased substantially at the level of early Stage 2 prolapse ( $1 \mathrm{~cm}$ above the hymen), with $67 \%$ answering yes to the question regarding bulge. The proportion of participants who felt that surgical outcome was less desirable also increased substantially at early Stage 2 prolapse $(1 \mathrm{~cm}$ above the hymen), with $52 \%$ describing that outcome as "not at all" or "somewhat" successful.

Conclusion: Early Stage 2 pelvic organ prolapse $(1 \mathrm{~cm}$ above the hymen $)$ is the anatomic threshold at which women identify both a vaginal bulge and a less desirable surgical outcome when they see what we see on examination.

Key Words: Prolapse, surgical outcomes 
Brief Summary Sentence: Early Stage 2 prolapse is the threshold at which women identify both a bulge and a less desirable surgical outcome when they see what we see. 


\section{Introduction}

Pelvic organ prolapse and surgical success after reconstructive surgery have evolved from a physician centered to a patient centered diagnosis based on clinical data from welldesigned epidemiologic studies [1-4]. Two of the more commonly used classification systems; Baden-Walker halfway system [5] and the Pelvic Organ Quantification (POP-Q) system [6] established "normalcy" as the absence of anatomical prolapse on physical examination by the health care provider. The NIH Panel on standardization of terminology for pelvic organ prolapse followed these recommendations by defining surgical success as the absence of anatomical prolapse on physical examination as well [7]. Surgical failure was evident when the leading edge of prolapse descended $1 \mathrm{~cm}$ above the hymen or beyond ( $\geq$ Stage II prolapse). Yet $37 \%$ of asymptomatic women ( $17 \%$ hysterectomized, median parity 2$)$ have Stage II prolapse suggesting incredibly high disease prevalence if these anatomic definitions are considered valid [2]. However, physical examination of nulliparous women reveals vaginal descent no greater than $1.46 \mathrm{~cm}$ above the hymen (<Stage II prolapse) [4] suggesting that pregnancy, multiparity, and hysterectomy may contribute to pelvic support defects in asymptomatic women regardless of the definition of prolapse or surgical success after reconstructive surgery [8]. Nonetheless, vaginal symptoms including the feeling of a vaginal bulge are the most reliable symptom of pelvic organ prolapse when the leading edge descends to or beyond the hymen [9]. Health outcomes researchers in female pelvic medicine and reconstructive surgery have redefined pelvic organ prolapse using this symptomatic threshold [1-3] rather than the previously established anatomic one. This new symptomatic threshold was used to retrospectively reclassify previously published surgical outcomes following vaginal reconstructive surgery 
resulting in an unprecedented improvement in success rates from $30-46 \%$ to $88 \%$ for the surgical team [10]. More recently, prospective studies have used a composite definition for surgical success (anatomic success with absence of symptoms or need for retreatment) creating two additional hurdles for the surgeon to clear, thereby reducing the probability for a successful health outcome even after the most durable repair [11].

Surgeons must reconcile what they see on pelvic examination after reconstructive surgery with what the patient feels especially when there is descent to the hymen in an asymptomatic patient. A finding of Stage II prolapse in an asymptomatic patient can lead to uncertainty for the surgeon and is partially based on a lack of knowledge about the clinical significance, natural history, or progression of "disease" in this population. Knowledge regarding the patient perspective of this problem is warranted, especially during the transition from a service based to a health outcomes based payment system, so reconstructive surgeons can provide guidance when legislative decisions are made.

We were interested in gaining the patient's perspective on a threshold for defining pelvic organ prolapse, and success/failure after reconstructive surgery "if they could see what we see" on physical examination.

\section{Materials and Methods}

We conducted a prospective, cross-sectional study of all eligible women presenting for a new patient visit to our tertiary Urogynecology referral center for care of any pelvic floor disorder. Women who declined participation, who were unable to consent for participation, or who were unable to complete study questionnaires in English were excluded. A convenience 
sample was approached and informed consent was obtained between August 2013 and August 2014. Study participation was completed prior to medical counseling to eliminate any possibility of information bias. This study was fully approved by the Institutional Review Board at Indiana University.

Participants were then assigned, by computerized block randomization, to see one of 4 videos. Each video contained the same six dynamic clips representative of various degrees of anterior vaginal wall support with Valsalva's maneuver in the supine position. The order of the clips was similarly randomized on the four videos. This double randomization was performed to minimize potential response bias introduced if prolapse stage was displayed by increasing or decreasing severity. The six clips represented Stage 0 POP, Stage 1 POP, early Stage 2 POP (leading edge $1 \mathrm{~cm}$ above the hymen), mid Stage 2 POP (leading edge at the hymen), late Stage 2 POP (leading edge $1 \mathrm{~cm}$ below the hymen), and Stage 3 POP, using the POP-Q staging system ${ }^{6}$. In light of the previously described importance of the hymen as a reference point for treatment success and failure, Stage 2 POP was sub-divided into varying degrees because it includes points of descent above and below the hymen. Figure 1 includes still pictures taken directly from the dynamic videos participants were asked to view. These images show the vaginal introitus with the posterior blade of a Grave's speculum compressing the posterior wall of the vagina and represent the maximal degree of descent of the anterior wall seen with Valsalva's maneuver in the supine position.

Immediately after viewing each clip, participants were prompted to answer three questions. They were first asked to give a Yes or No response to the following question: "In your 
opinion, does this patient have a bulge or something falling out that she can see or feel in the vaginal area". This question was adapted from question 3 of the pelvic organ prolapse distress inventory (POP-DI) subdomain of the validated Pelvic Floor Distress Inventory (PFDI-20) short form [12-13]. They were then asked to rate the certainty of their response on a $10 \mathrm{~cm}$ Visual Analog Scale, where 0 represented "Absolutely Uncertain" and 10 represented "Absolutely Certain". This was ultimately converted to percentage certainty. Finally, participants were asked to give their opinion on surgical outcome on a 4-point Likert scale [11]. They were asked: "Assume this patient has previously had surgery to treat her vaginal bulge. In your opinion, has her surgery been Very Successful Moderately Successful Somewhat Successful

Not at all Successful." Although no specific clinical context was provided to participants, all had bladder, vaginal, or bowel symptoms that warranted Urogynecology referral, mimicking the experience of patients with a number of pelvic floor disorders who are presented with the PFDI-20 and PFIQ-7 prior to their subspecialty physician visit.

Additional demographic and clinical variables including age, race, chief complaint, and overall prolapse stage were then collected from the medical record. In addition, scores on two condition specific validated questionnaires routinely administered in our office, the Pelvic Floor Distress Inventory (PFDI-20) and the Pelvic Floor Impact Questionnaire (PFIQ-7), were collected. Socioeconomic status (SES) was measured using the Hollingshead Four Factor Index [14]. This is a composite index utilizing three domains to measure socioeconomic status, including marital status, educational attainment, and occupation. The range on this scale is 8-66 with higher scores indicating higher SES. Finally, dispositional optimism was measured via the Life Orientation Test (LOT) [15]. This self-administered questionnaire was developed to assess 
individual differences in generalized optimism versus pessimism with LOT scores frequently used to assess the health consequences of this personality variable trait. The range on this scale is 0-24 with higher scores indicating more dispositional optimism rather than pessimism.

Descriptive statistics were performed as appropriate for categorical, as well as continuous variables based on review of their histograms. A Cochran's $Q$ test was performed to test the null hypothesis that there was no difference in K-related population proportions that identified a "symptomatic" vaginal bulge in each of the 6 video clips. Mann-Whitney $U$ tests were performed to test the null hypothesis that participant's degree of response certainty about the presence or absence of a "symptomatic" vaginal bulge did not differ at each prolapse stage on the 6 video clips. Finally, a Cochran's $Q$ test was performed to test the null hypothesis that there was no difference in k-related population proportions that felt surgical outcome was less desirable in each of the 6 video clips. We dichotomized anatomic thresholds into two groups; (1) at or above the hymen, and (2) below the hymen to determine when study participants first identified a "symptomatic" vaginal bulge. We dichotomized surgical outcome into two groups; (1) desirable (Very and Moderately Successful), and (2) less desirable (Somewhat and Not at all Successful) to determine at what anatomic threshold study participants first identified a less desirable surgical outcome. Bivariate analyses was performed to determine if any baseline characteristics were predictive of a patient centered anatomic threshold for defining a vaginal bulge or surgical success/failure either at and above or below the hymen. Statistical analysis was performed using SPSS V21.0 (IBM Corp, Armonk, NY). P values of $<0.05$ were considered statistically significant. 


\section{Results}

A total of two hundred women were randomized during the study period and 197 women completed the study questionnaires. The mean age of participants was 60 years (SD $13.5)$ and the majority of participants were white (92\%). Forty-nine percent (49\%) of participants presented with a chief complaint of POP and 35\% presented with urinary incontinence or overactive bladder. The majority of women had never undergone prior incontinence or prolapse surgery ( $85 \%$ and $86 \%$ respectively). The majority of women had $\leq$ Stage 2 pelvic organ prolapse on physical examination (72\%). Mean SES score, as calculated from the Hollingshead Four Factor Index (scale 8-66), was 43 (SD 12), consistent with the social strata of medium sized business owners, technical workers, and minor professionals. Mean LOT score, as calculated from the Life Orientation Test (scale 0-24), was 17 (SD 5) indicating moderate optimism. Briefly explained, our study participants tended to be more generally optimistic than pessimistic. Demographic and clinical characteristics are summarized in Table 1.

The proportion of participants who identified the presence of a symptomatic bulge increased substantially at the level of early Stage 2 POP $(1 \mathrm{~cm}$ above the hymen) $(p<0.001)$. Sixty-seven percent (67\%) of participants answered yes to the question "In your opinion, does this patient have a bulge or something falling out that she can see or feel in the vaginal area" after viewing the video clip representative of this stage of anterior vaginal wall support. This is a relatively sharp departure from the responses to the same question after viewing the Stage 1 POP clip, where only $38 \%$ of participants responded affirmatively. Thus, $1 \mathrm{~cm}$ above the hymen 
seems to be the anatomic threshold at which women identified a vaginal bulge when they were allowed to see what we see on examination. Figure 2

A graph of the degree of response certainty about the presence and absence of a bulge intersects at Stage 1 prolapse where an equal level of uncertainty or "complete uncertainty" of participant responses exists. Immediately adjacent to Stage I prolapse there is a clear divergence of the two graphs again supporting $-1 \mathrm{~cm}$ above the hymen as the patient centered anatomic threshold for "symptomatic" vaginal bulge identification when they can see what we see, in a statistically significant manner. The graphs do converge again, to a minor degree, at Mid Stage II POP (the hymen). This could be related to factors other than the bulge affecting participant responses. It could also be related to the number of participants in the study and a lack of statistical power. Figure 3

The proportion of participants who felt that surgical outcome was less desirable also increased substantially at early Stage 2 POP $(1 \mathrm{~cm}$ above the hymen) $(p<0.001)$. Fifty-two percent (52\%) of participants described the surgical outcome as "not at all successful" or "somewhat successful" (less desirable outcome) on a 4-point Likert scale after watching the video clip representative of this stage of anterior vaginal wall support. This is also a relatively sharp departure from the responses to the same question after viewing the Stage 1 POP clip, where only $38 \%$ of participants rated the surgical outcome as "not at all successful" or "somewhat successful". Thus, $1 \mathrm{~cm}$ above the hymen may also be the anatomic threshold at which women identify surgical failure when they are allowed to see what we see on examination. Figure 4 
Notably, $90 \%$ of participants first identified symptomatic bulge as occurring at some level at or above the hymen after video viewing. Only $10 \%$ felt that symptomatic bulge did not occur until the prolapse extended beyond the hymen. In addition, $84 \%$ first identified a less desirable surgical outcome as prolapse occurring at some level at or above the hymen while only $16 \%$ felt that a less desirable surgical outcome did not occur until the prolapse extended beyond the hymen. Figure 5. No single demographic or clinical characteristic predicted whether "symptomatic" vaginal bulge, or less desirable surgical outcome was first identified with support at and above, or below the hymen on bivariate analysis. This includes age, POP-Q stage on exam, or PFDI and PFIQ scores.

\section{Discussion}

Barber et al recommended the use of patient perspective by including absence of bulge symptoms in any definition of surgical success after pelvic organ prolapse surgery, with the hymen as a reasonable threshold, until we have an understanding of the clinical significance of asymptomatic stage II disease [11]. However, the vaginal hymen, is located in an inaccessible region for visual anatomic inspection and disease identification by the patient. The short form PFDI-20 and PFIQ-7 are psychometrically valid, reliable, and responsive to change in women with pelvic floor disorders for assessment of symptom bother and impact on activities of daily living, respectively [12-13]. Of the 41 total questions that make up both questionnaires, only item number 3 in the POP-DI sub domain of the PFDI-20 asks patients if they "usually have a bulge or something falling out that they can see or feel in the vaginal area." Because the vagina is located in a less anatomically accessible region for visual inspection, a yes response to 
question 3 is highly correlated with self-identification of a vaginal bulge at or beyond the hymen, once the bulge can be seen. However, a no response should not rule out the existence of disease from the patient's and provider's perspective.

We purposely used the same wording from question 3 in the POP-DI subdomain of the PFDI-20 to gain the patients perspective on a diagnosis of pelvic organ prolapse when "they saw what we saw" after video presentation. Similarly, we purposely used the same wording from the global impression of success scale to gain the patient's perspective on surgical success when "they saw what we saw" after video presentation [11]. In each case we found that patients considered early $(-1 \mathrm{~cm})$ Stage II disease clinically significant in their definition of pelvic organ prolapse and surgical success after reconstructive surgery. Anatomic thresholds of one centimeter above the hymen and "at or beyond the hymen" can coexist in the field of female pelvic medicine and reconstructive surgery. A diagnosis of pelvic organ prolapse based on a leading edge of -1cm should not and would not require surgery, it would be "non-operable", until it descends beyond the hymen, when it becomes symptomatic, and "operable." To be clear, we do not advocate, nor do we feel that these findings support, surgical intervention on asymptomatic women with descent to -1 . Regardless, it appears that women, at least in the context of this study, identified descent to this level as less than ideal anatomical support.

We understand that this reasoning increases the percentage of "asymptomatic" women with disease from $3 \%$ [16] to $37-69.8 \%$ [4] based solely on an anatomic landmark. Yet this anatomic threshold $(-1)$ recognizes the contribution of pregnancy and vaginal delivery on pelvic support in multiparous women $[2,4]$. Furthermore, we believe that this anatomic threshold 
does have utility for assessment of surgical outcome after prolapse repair. Appraisal of surgical outcome continues to be the driving force for innovations in the field including mid-urethral slings, ultra-lightweight macroporous mesh, biologic scaffolds and grafts, and minimally invasive approaches to abdomino-pelvic reconstructive surgeries.

There are several limitations to this study that must be considered before our conclusions can be accepted as valid. First, we did not establish the content validity or interobserver reliability of our video presentations for each POP-Q prolapse stage through consensus amongst experts in the field of female pelvic medicine and reconstructive surgery. In addition, we were unable to standardize the appearance of the video models' external genitalia (age, BMI etc.) due to limitations of the available videos. However, the senior author who has over 20 years of experience in the field established the content validity of our video presentations while we minimized systematic error through the randomization process. The clip sequence demonstrating POP-Q prolapse stage was randomly ordered in each of the four videos which were randomly watched by study participants. Nonetheless, the results of our study may have differed if the videos containing clips of POP-Q prolapse stage were chosen by a panel of experts who were uninvolved in study design. Secondly, we provided limited clinical context for participants other than the study questions and the informed consent documents prior to video viewing. We purposely designed the study to mimic the experience of patients with a number of pelvic floor disorders who are presented with the PFDI-20 and PFIQ-7 prior to their subspecialty physician visit. Again, our study results may have differed if we limited study participation to women with pelvic organ prolapse who were presented with greater clinical context about their disorder prior to video viewing. Finally, our study was conducted with 
participants primarily affected by pelvic floor disorders and recruited from a Urogynecology center. Thus, they may not be generalizable to women at large.

Study participants identified a leading edge descending one centimeter short of the hymen as the anatomic threshold for a "symptomatic" vaginal bulge suggestive of pelvic organ prolapse after seeing what we see on physical examination. We recommend consideration of the following possible definitions of pelvic organ prolapse and surgical success/failure. "Nonoperable" pelvic organ prolapse is a vaginal bulge whose leading edge descends beyond $1 \mathrm{~cm}$ above the hymen with strain in the supine position in an asymptomatic patient. "Operable" pelvic organ prolapse is a vaginal bulge whose leading edge descends beyond the hymen when symptoms are likely to occur. Study participants who characterize surgeries as unsuccessful when the leading edge of prolapse descends beyond $1 \mathrm{~cm}$ above the hymen validate providers feelings "when they see what we see on physical examination" because of our lack of knowledge about the natural history, or progression of "asymptomatic" disease. Yet the provider and patient are likely willing to accept this degree of descent as "non-operable" pelvic organ prolapse because it remains asymptomatic. While we accept the fact that the objective of reconstructive surgery is to relieve symptoms through bulge reduction we are unwilling to characterize a surgical outcome as successful when in the eyes of both the patient and provider there is anatomic descent of the leading edge from $-1 \mathrm{~cm}$ to the hymen on examination.

\section{References}


1. Swift SE, Tate SB, Nicholas J. Correlation of symptoms with degree of pelvic organ support in a general population of women: What is pelvic organ prolapse? Am J Obstet Gynecol. 2003;189:372-377.

2. Swift S, Woodman P, O'Boyle A, et al. Pelvic Organ Support Study (POSST): the distribution, clinical definition, and epidemiologic condition of pelvic organ support defects. Am J Obstet Gynecol 2005;192:795-806.

3. Tan JS, Lukacz ES, Menefee SA, et al. Predictive value of prolapse symptoms: a large database study. Int Urogynecol J Pelvic Floor Dysfunct 2005;16: 203-9.

4. Trowbridge ER, Fultz NH, Patel DA, et al. Distribution of pelvic organ support measures in a population-based sample of middle-aged, community-dwelling African American and white women in southeastern Michigan. Am J Obstet Gynecol 2008;198:548.e1548.e6.

5. Baden WF, Walker T. Fundamentals, Symptoms, and Classification. In: Baden WF, Walker T, eds. Surgical Repair of Vaginal Defects. Philadelphia, PA: JB Lippincott Company; 1992:9-23.

6. Bump RC, Mattiasson A, Bo K, Brubaker LP, et al. The standardization of terminology of female pelvic organ prolapse and pelvic floor dysfunction. Am J Obstet Gynecol 1996;175:10-7.

7. Weber AM, Abrams P, Brubaker L, et al. The standardization of terminology for researchers in female pelvic floor disorders. Int Urogynecol J Pelvic Floor Dysfunct $2001 ; 12: 178-86$ 
8. Harris RL, Cundiff GW, Coates KW, et al. Urinary Incontinence and Pelvic Organ Prolapse in Nulliparous Women. Obstet Gynecol. 1998:92(6);951-954

9. Barber M. Symptoms and Outcome Measures of Pelvic Organ Prolapse. Clin Obstet \& Gynecol. 2005;48(3):648-661

10. Chmielewski L, Walters MD, Weber AM, Barber MD. Reanalysis of a randomized trial of 3 techniques of anterior colporrhaphy using clinically relevant definitions of success. Am J Obstet Gynecol 2011;205:69.e1-8

11. Barber M, Brubaker I, Nygaard I, et al. Defining Success after Surgery for Pelvic Organ Prolapse. Obstet Gynecol 2009;114:600-9

12. Barber MD, Kuchibhatla MN, Pieper CF, et al. Psychometric evaluation of 2 comprehensive condition-specific quality of life instruments for women with pelvic floor disorders. Am J Obstet Gynecol 2001;185:1388-95.

13. Barber MD, Walters MD, Bump RC. Short forms of two condition specific quality of life questionnaires for women with pelvic floor disorders (PFDI-20 and PFIQ-7). Am J Obstet Gynecol. 2005;193:103-113.

14. Hollingshead, AB. Four-factor index of social status. Unpublished manuscript, Yale University, New Haven, CT. 1975.

15. Glaesmer H, Rief W, Martin A, et al. Psychometric properties and population-based norms of the Life Orientation Test Revised (LOT-R). Br J Health Psychol. 2012:17;432445.

16. Nygaard I, Barber M, Burgio K, et al. Prevalence of Symptomatic Pelvic Floor Disorders in US Women. JAMA 2008;300(11): 1311-1316 


\section{Figure Legend}

Figure 1. Still images taken directly from the dynamic videos and representing the maximal degree of descent of the anterior wall seen in each clip

Figure 2. Identification of the presence of bulge by study participants after viewing video clips representative of various stages of anterior vaginal wall support

Figure 3. Degree of certainty of study participants of the presence or absence of a vaginal bulge after viewing video clips representative of various stages of anterior vaginal wall support 
Figure 4. Identification of surgical outcome by study participants after viewing video clips representative of various stages of anterior vaginal wall support

Figure 5. Participants dichotomized by identification of symptomatic bulge and less desirable surgical outcome at/above or below the hymen 